\title{
Clinical utility of microRNA-378 as early diagnostic biomarker of human cancers: a meta-analysis of diagnostic test
}

\author{
Zhan-Zhan Li ${ }^{1}$, Liang-Fang Shen ${ }^{1}$, Yan-Yan Li ${ }^{2}$, Peng Chen ${ }^{3}$, Li-Zhang Chen ${ }^{4}$ \\ ${ }^{1}$ Department of Oncology, Xiangya Hospital, Central South University, Changsha, Hunan Province 410008, China \\ ${ }^{2}$ Department of Nursing, Xiangya Hospital, Central South University, Changsha, Hunan Province 410008, China \\ ${ }^{3}$ Department of Orthopedics, Xiangya Hospital, Central South University, Changsha, Hunan Province 410008, China \\ ${ }^{4}$ Department of Epidemiology and Health Statistics, School of Public Health, Central South University, Changsha, Hunan \\ Province 410078, China
}

Correspondence to: Li-Zhang Chen, email: liche4005@126.com

Keywords: cancer, microRNA-378, early diagnosis, meta-analysis, tumor marker

Received: April 12, $2016 \quad$ Accepted: June 06, $2016 \quad$ Published: July 19, 2016

\section{ABSTRACT}

A meta-analysis was performed to evaluate the diagnostic value of miR-378 for detecting human cancers. Systematic electronic searches were conducted in PubMed, Web of Science, Embase, China National Knowledge Infrastructure, and Wanfang from the inception to January 15, 2016. We used the bivariate mixed effects models to estimate the combined sensitivity, specificity, PLRs (positive likelihood ratios), NLR (negative likelihood ratios), DORs (diagnostic odds ratios) and their $95 \%$ CI (confidence intervals) for assessing the diagnostic performance of miR-378 for cancers. Twelve studies were included in the meta-analysis, with a total number of 1172 cancer patients and 809 health controls. The overall estimated sensitivity and specificity were 0.75 and 0.74. The pooled PLR was 2.91, NLR was 0.34, DOR was 8.50, and AUC (Area Under the Curve) was 0.81 . The subgroup analyses suggested that AUC for plasma-based is higher than serum-based. The overall diagnostic values of miR-378 in the present meta-analyses are moderate accurate for human cancers; The source of specimen has an effect on the diagnostic accuracy. The diagnostic value of serum-based was higher than that of plasma-based.

\section{INTRODUCTION}

Cancers have become an important public issue on a world scale. According to the World Cancer Research Fund report in 2014, the newly added cancer patients had 1400 million worldwide [1]. Previous studies had suggested that many reasons could lead to cancer occurrence such as unhealthy lifestyles, environment exposing, and special eating habitats [2-4]. With the rising mortality caused by cancers, the prognosis and survival situation of the cancer patients are not optimistic. It was reported that the five-year survival rate for cancer patients was about 50\% [5]. But the 5-year survival rate for cancer patients after diagnosis and treatments strongly depends on the types of cancer. Patients with prostate cancer have a more than $80 \%$ chance of survival past 5 years while the 5 -year survival rate for pancreatic patients is less than $6 \%$ $[6,7]$. Some other cancers also have low 5-year survival rates such as $5-9 \%$ for hepatocellular carcinoma patients [8], 13\% for lung cancer patients [9], and $9.8 \%$ for patients with central nervous systems tumors [10].

Successful treatment of cancer patients, which largely depends on early detection, is important to improve survival rates and life qualities of patients with cancers. At present, the most common method to diagnosis cancers is histopathological examination. However, the invasive procedure restricts its application [11]. The markers examination of circulating blood in detecting cancers (serum/plasma) received widespread attention because of its less trauma and acceptability for cancer patients. The $\alpha$-fetoprotein (AFP), carcinoembryonic antigen (CEA), and carbohydrate antigen (CA) are the most commonly used serum markers for early detection of cancers. These biomarkers do not meet the requirements of clinical practice and population screening because of their low sensitivity or specificity and expenses, especially for a wide 
screening for national level population [12]. Therefore, a simple, quick and sufficient marker for detecting cancers are urgently needed. MicroRNAs or miRNAs, non-coding RNAS with 17-25 nucleotides, are small endogenous RNAs. The miRNAs are found in a variety of biological cells and evolutionarily conserved [13]. It is suggested that the high or low expression miRNAs are detected in different kinds of human cancers, the expression profiles and levels exhibit an apparent tissue specificity and time phases, and miRNAs can resist enzyme degradation. The most importance is that miRNAs also can stably exist in the circulating blood because of their particular structure [14], which are ideal biomarkers of detecting cancers. The miRNA-378 is an important tumor-related gene regulatory site. Previous study found that the miRNA-378 expression levels could identify cancer patients and health individuals [15]. However, the diagnostic values of miRNA-378 remain inconsistent in different studies, which could be caused by the limitation of sample size, study group and cancers types [16, 17]. We conducted a meta-analysis to evaluate the diagnostic values of miRNA-378 for detecting human cancers.

\section{RESULTS}

\section{Study selection}

Figure 1 shows the process of literature search and screening. The initial search returned 836 records and 2 records were obtained through manual retrieval. 429 records were left for further screening after removing the duplicated records. After reviewing abstracts and titles, we excluded 368 records and 61 articles were thought to be potentially eligible for inclusion. We excluded 49 records because the following reasons: 33 records with unrelated to diagnostic values or other miRNAs, and 16 insufficient data. Finally, 12 studies were included in the meta-analysis [16-27]. One of them included four groups of data [25].

\section{Study characteristics}

The Table 1 summarized the main characteristics of the included studies. These studies were published from 2013 to 2015. The sample sizes ranged from 42 to 295, with a total number of 1172 cancer patients and 809 health controls. In the present studies, the types of cancers included renal cell cancer (RCC), gastric cancer (GC), nasopharyngeal carcinoma $(\mathrm{NC})$, colorectal carcinoma (CC). breast cancer (BC), pancreatic cancer(PC). Among the 12 studies, 7 studies were conducted in Asian population, and 5 studies were from Caucasian population. Six studies samples were from serum, six were from plasma, and two were from tissues. All miRNAs were detected through quantificational real-time polymerase chain reaction (qRT-PCR).

\section{Assessment of quality}

The overview of the quality of included were presented in Supplementary Figure S1 and Supplementary Figure S2. Four studies given unclear description for patient selection. The index test was judged as unclear in six studies that did not confirm whether the results were interpreted without knowledge of the results of the reference standard as well as reference standard. Five studies without avoided case-control design, inappropriate exclusions or interpreted results with knowledge of the results of the references standard were considered as having a high risk bias. For applicability concerns, each of three domain keys has one study that given unclear description.

\section{Pooled diagnostic values}

The Spearman coefficient was -0.082 and $P=0.770$, which means no threshold effect. The $I^{2}$ values for sensitivity and specificity were more than $50 \%$, and random effect models were used. The estimated diagnostic values of miR-378 for detecting cancers are shown in Table 2 . The overall estimated sensitivity and specificity were 0.75 (95\% CI: 0.71-0.78) and 0.74 (95\% CI: 0.690.79). The pooled PLR was 2.91 (95\% CI:2.38-3.55), NLR was 0.34 (95\% CI:0.29-0.41), and DOR was 8.50 (95\% CI: 6.01-12.01). Fangan plot was shown in Figure 2. The overall SROC curve was shown in Figure 2, Figure 3 and AUC was 0.81 (95\% CI: 0.77-0.84). The diagnostic accuracy of miR-378 for cancers was relatively high.

\section{Subgroup analyses}

Subgroup analyses were conducted in the ethnicity (Asian vs Caucasian), cancer type (renal cell carcinoma vs other types), sample types (serum-based, plasma-based and) setting. The subgroup results of all estimates were presented in Table 2 (sensitivity, specificity, PLR, NLR, and DOR). There are no obvious differences between combined results in the ethnicity and cancer type. The pooled sensitivity and specificity and AUC were similar (Table 2). The results suggested that ethnicity may be not an influence factor on heterogeneity. However, the sample types indicated significant difference of estimated results. The AUC for plasma-based $(0.80,0.77-0.84)$ is significantly different from serum-based $(0.70,0.66-0.74)$ in Figure 4. The diagnostic accuracy of serum-based is higher than that of plasma-based. It suggests that the diagnostic cutoff values of miR-378 for detecting cancers is correlate to source of samples. The rest results were 
similar. The pooled sensitivity, specificity, PLR, and NLR for serum-based were: 0.75 (95\% CI: $0.71-0.79), 0.74$ (95\% CI: 0.68-0.80), 2.91 (95\% CI: 2.22-3.81), 0.34 (95\% CI: 0.27-0.42); For plasma-based: 0.68 (95\% CI: 0.62-0.74), 0.72 (95\% CI: 0.59-0.81), 2.38 (95\% CI: $1.59-3.55)$, and 0.44 (95\% CI: $0.34-0.58)$.

\section{Sensitivity analyses and publication bias}

We conducted sensitivity analyses through sequentially excluding individual studies, and the summary sensitivity and specificity, PLR, NLR and ACU were altered (data were not given), indicating that the present pooled estimated were stable. We used Deek's plot to evaluate the publication bias. The bias test shown no existence of publication bias $(t=0.09, P=0.929)$ as indicated in Figure 5.

\section{DISCUSSION}

In the present meta-analysis, twelve studies were included, and the pooled results including all studies showed miRNA-378 gave an AUC of 0.81 (95\% CI:0.770.84 ) with a sensitivity value of $75 \%$ and specificity of $74 \%$ in identifying the patients with cancers from health individuals. The miRNA-378 showed a moderate accuracy in detecting cancer patients. We also found the source of specimen had an effect on the diagnostic value of
miRNa-378. The specimen of serum-based is significantly higher than plasma-based specimen (AUC: 0.80, 0.770.84 vs $0.70,0.66-0.74)$. The higher ACU value means better diagnostic ability of balance between sensitivity and specificity, especially for renal cell carcinoma.

The pooled diagnostic value of miR-378 is higher than traditional clinical markers such as CEA and CA19-9 [28], indicating the relatively high accuracy of miRNA-378 as an early diagnosing biomarker of cancers. Our results are almost equal to previous several miRNAs. Shen et al. conducted a meta-analysis of miRNA-21 in detecting human cancers. This study yielded a AUC of 0.88 with a sensitivity of $78 \%$ and a specificity of $83 \%$ for all cancers, and this study did not give the diagnostic value of RCC [29]. Another study conducted by Tang used different methods to evaluate the diagnostic value of all miRNAs for RCC. They used the hazard ratio to quantify the criteria of miRNAs instead of the sensitivity and specificity. The five miRNAs provided a reliable tool for RCC patients, especially for clear cell RCC [30]. Our results found that miRNA-378 had a AUC of 0.81 as well as other cancer types of the present results. The diagnostics value was similar to the oral squamous cell carcinoma (AUC of 0.832). This study did not focus on single miRNA but all miRNAs and gave an integrated miRNA expression profiling analysis [31]. The highest AUC in all included studies is 0.86 with $87.5 \%$ sensitivity and $70.73 \%$ specificity in diagnosing the GC patients [20],

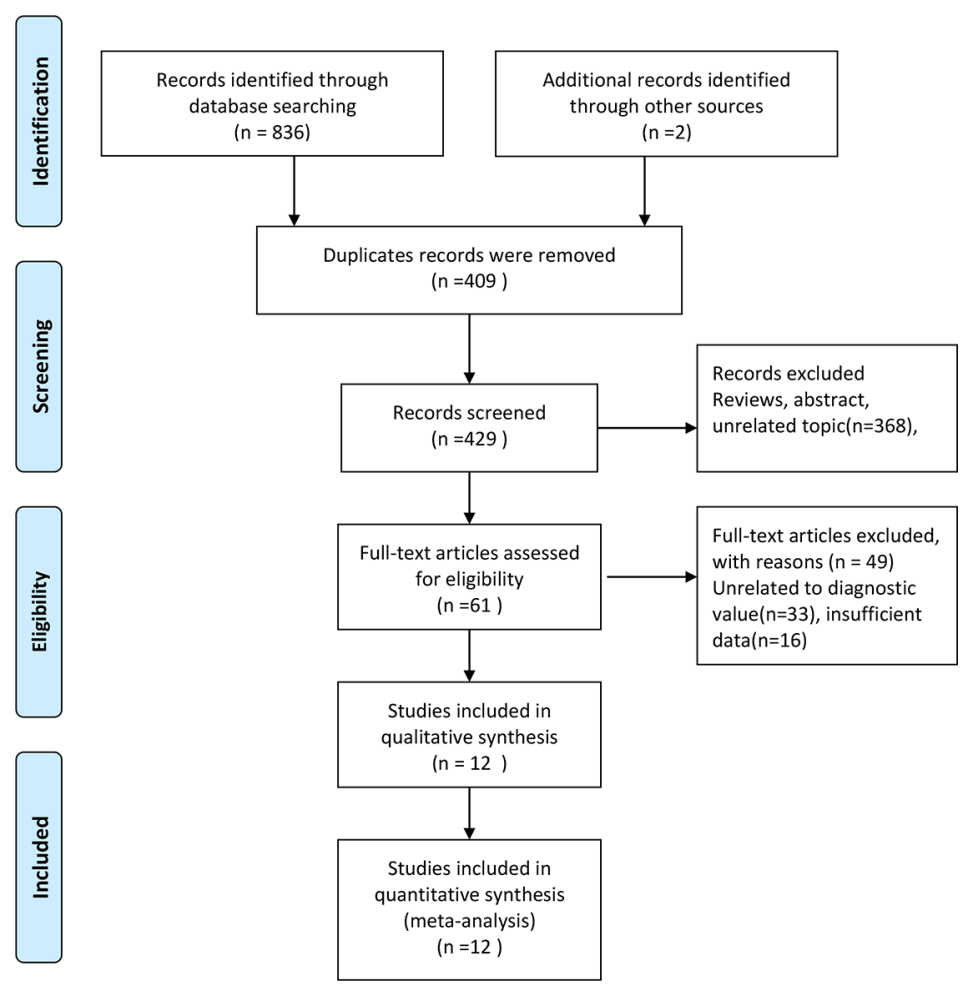

Figure 1: Flow diagram of studies selection process. 
Table 1: Characteristics of the included studies in the meta-analysis of miR-378 for cancer detection Author Year Country Ethnicity Case Control Type Sample $\begin{gathered}\text { Methods of } \\ \text { detection }\end{gathered}$ TP $\quad$ FP FN TN

\begin{tabular}{|c|c|c|c|c|c|c|c|c|c|c|c|c|}
\hline Redova & 2012 & Germany & Caucasian & 90 & 35 & RCC & Serum & qRT-PCR & 63 & 14 & 27 & 21 \\
\hline Peng & 2015 & China & Asian & 32 & 32 & $\mathrm{HCC}$ & Tissue & qRT-PCR & 29 & 8 & 2 & 23 \\
\hline Liu & 2012 & China & Asian & 61 & 61 & GC & Serum & qRT-PCR & 53 & 18 & 8 & 43 \\
\hline Zanutto & 2014 & Italy & Caucasian & 29 & 29 & $\mathrm{CC}$ & Plasma & qRT-PCR & 23 & 8 & 6 & 21 \\
\hline Liu & 2013 & China & Asian & 217 & 73 & $\mathrm{NC}$ & Plasma & qRT-PCR & 146 & 29 & 71 & 44 \\
\hline Yin & 2014 & China & Asian & 101 & 40 & $\mathrm{BC}$ & Tissue & qRT-PCR & 69 & 4 & 32 & 36 \\
\hline Fedorko & 2015 & Czech & Caucasian & 195 & 100 & $\mathrm{RCC}$ & Serum & qRT-PCR & 159 & 17 & 36 & 83 \\
\hline $\mathrm{Li}$ & 2013 & America & Caucasian & 41 & 19 & $\mathrm{PC}$ & Serum & qRT-PCR & 31 & 4 & 10 & 15 \\
\hline Hauser & 2012 & Germany & Caucasian & 25 & 25 & $\mathrm{RCC}$ & Serum & qRT-PCR & 18 & 16 & 7 & 19 \\
\hline Wang & 2015 & China & Asian & 107 & 107 & $\mathrm{RCC}$ & Serum & qRT-PCR & 79 & 28 & 26 & 81 \\
\hline Wang $^{\mathrm{a}}$ & 2015 & China & Asian & 28 & 28 & $\mathrm{RCC}$ & Serum & qRT-PCR & 20 & 8 & 7 & 21 \\
\hline Wang $^{\mathrm{b}}$ & 2015 & China & Asian & 79 & 79 & $\mathrm{RCC}$ & Serum & qRT-PCR & 63 & 16 & 21 & 58 \\
\hline Wang ${ }^{\mathrm{c}}$ & 2015 & China & Asian & 76 & 107 & RCC & Serum & qRT-PCR & 54 & 22 & 21 & 86 \\
\hline $\mathrm{Li}$ & 2015 & China & Asian & 22 & 20 & $\mathrm{RCC}$ & Plasma & qRT-PCR & 15 & 2 & 7 & 18 \\
\hline $\mathrm{Li}$ & 2013 & China & Asian & 69 & 54 & $\mathrm{GC}$ & Plasma & qRT-PCR & 45 & 13 & 24 & 32 \\
\hline
\end{tabular}

RCC, renal cell carcinoma; HCC, hepatocellular carcinoma; CC, colorectal carcinoma; $\mathrm{BC}$, breast cancer; PC, pancreatic cancer; NC, nasopharyngeal carcinoma; GC, gastric cancer; qRT-PCR, quantificational real-time polymerase chain reaction.

and the lowest AUC is 0.66 (95\% CI: 0.57-0.77) for GC [27]. There are only two studies reporting the diagnostic value of GC, and further studies are needed.

miRNA-378 could promote the expression of protooncogenes through targeted localization and inhibiting the BTG (B-cell translocation gene) prohibiting. In addition, miR-378 could be the downstream targeted site of the c-Myc oncoprotein, which was involved in stable transfection of miR-378 resulted in cell survival, tumor growth and angiogenesis [32]. These possible mechanisms make miRNA-378 become a potential biomarker of detecting cancers. In the present meta-analysis, eight studies of the levels of miRNA-378 in the serum of RCC patients are included in the meta-analysis. Redoval found that the level of miRNA-378 increased in serum of RCC patients compared to healthy controls. miRNA-378 also 
Table 2: Summary estimated of diagnostic performance of miR-378 for cancer detection

\begin{tabular}{|c|c|c|c|c|c|c|c|}
\hline Category & Cases/controls & SEN (95\% CI) & SPE $(95 \%$ CI) & PLR (95\% CI) & NLR (95\% CI) & DOR $(95 \%$ CI) & $\operatorname{AUC}(95 \% \mathrm{CI})$ \\
\hline Overall & $1172 / 809$ & $0.75[0.71-0.78]$ & $0.74[0.69-0.79]$ & $2.91[2.38-3.55]$ & $0.34[0.29-0.41]$ & $8.50[6.01-12.01]$ & $0.81[0.77-0.84]$ \\
\hline \multicolumn{8}{|l|}{ Ethnicity } \\
\hline Asian & $792 / 601$ & $0.74[0.69-0.79]$ & $0.76[0.70-0.80]$ & $3.02[2.44-3.75]$ & $0.35[0.28-0.42]$ & $8.77[6.01-12.80]$ & $0.81[0.78-.84]$ \\
\hline Caucasian & $380 / 208$ & $0.76[0.69-0.81]$ & $0.71[0.59-0.80]$ & $2.60[1.72-3.94]$ & $0.34[0.24-0.48]$ & $7.62[3.63-16.03]$ & $0.80[0.76-0.83]$ \\
\hline \multicolumn{8}{|l|}{ Cancer Type } \\
\hline Renal cell carcinoma & $622 / 501$ & $0.75[0.71-0.78]$ & $0.74[0.69-0.79]$ & $2.91[2.38-3.55]$ & $0.34[0.29-0.41]$ & $8.50[6.01-12.01]$ & $0.81[0.77-0.86]$ \\
\hline Other types & $550 / 308$ & $0.76[0.68-0.83]$ & $0.73[0.66-0.8]$ & $2.87[2.13-3.85]$ & $0.32[0.23-0.46]$ & 8.89 [4.95-15.92] & $0.81[0.77-0.84]$ \\
\hline \multicolumn{8}{|l|}{ Sample types } \\
\hline Serum-based & $702 / 561$ & $0.75[0.71-0.79]$ & $0.74[0.68-0.80]$ & $2.91[2.22-3.81]$ & $0.34[0.27-0.42]$ & $8.65[5.45-13.73]$ & $0.80[0.77-0.84]$ \\
\hline Plasma-based & $337 / 176$ & $0.68[0.62-0.74]$ & $0.72[0.59-0.81]$ & $2.38[1.59-3.55]$ & $0.44[0.34-0.58]$ & $5.31[2.84-10.13]$ & $0.70[0.66-0.74]$ \\
\hline
\end{tabular}

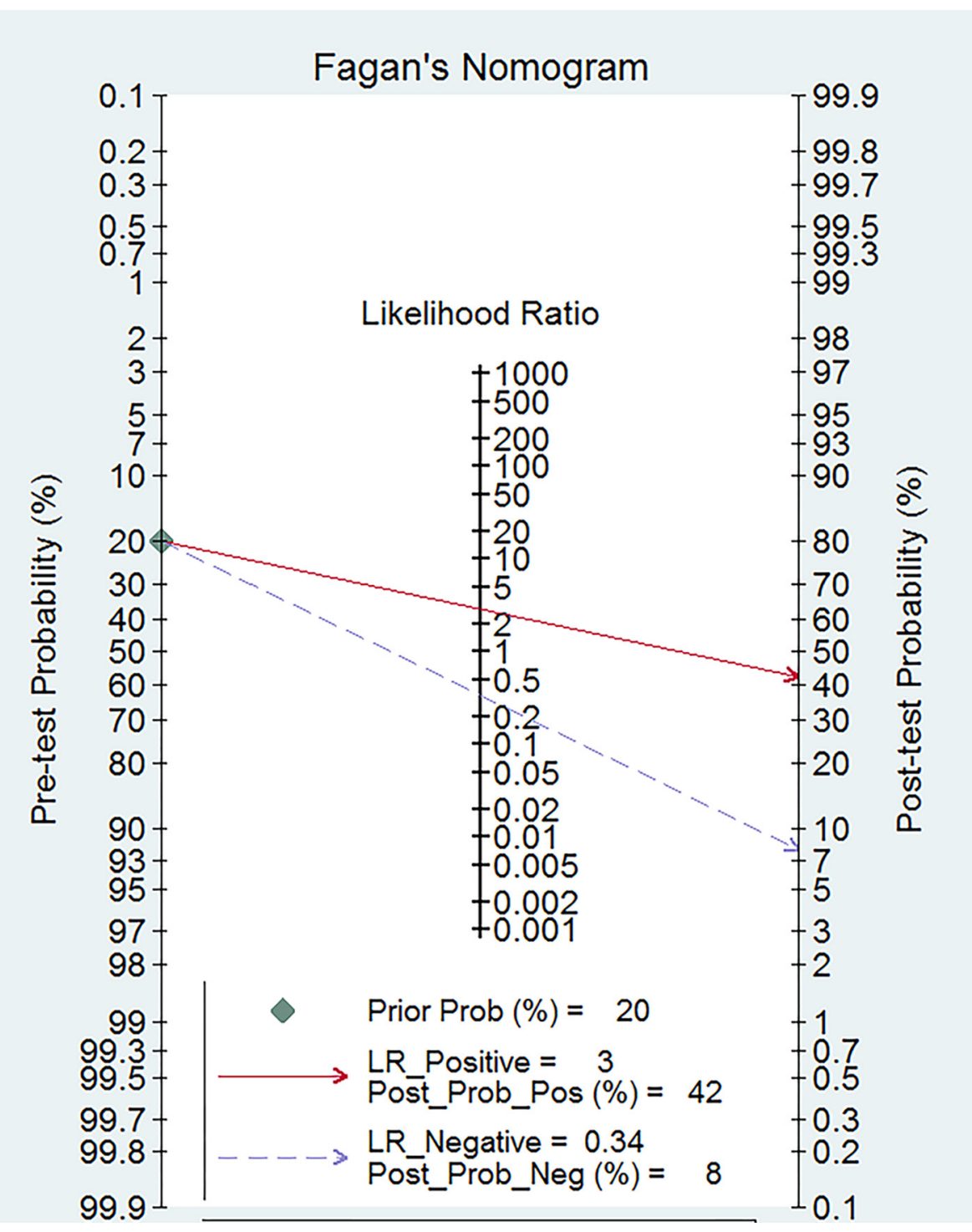

Figure 2: Fagan diagram evaluating the overall diagnostic value of miR-378 for cancer. 


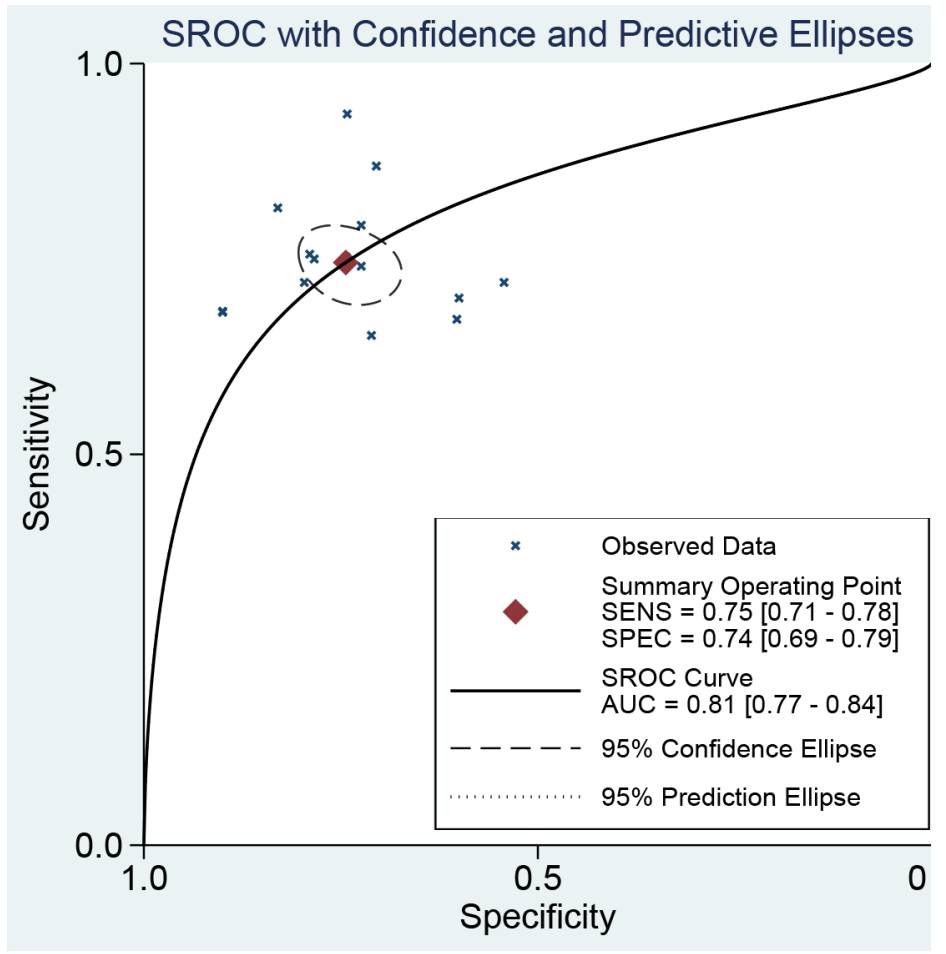

Figure 3: The SROC curve of miR-378 test for the diagnosis of various cancers.
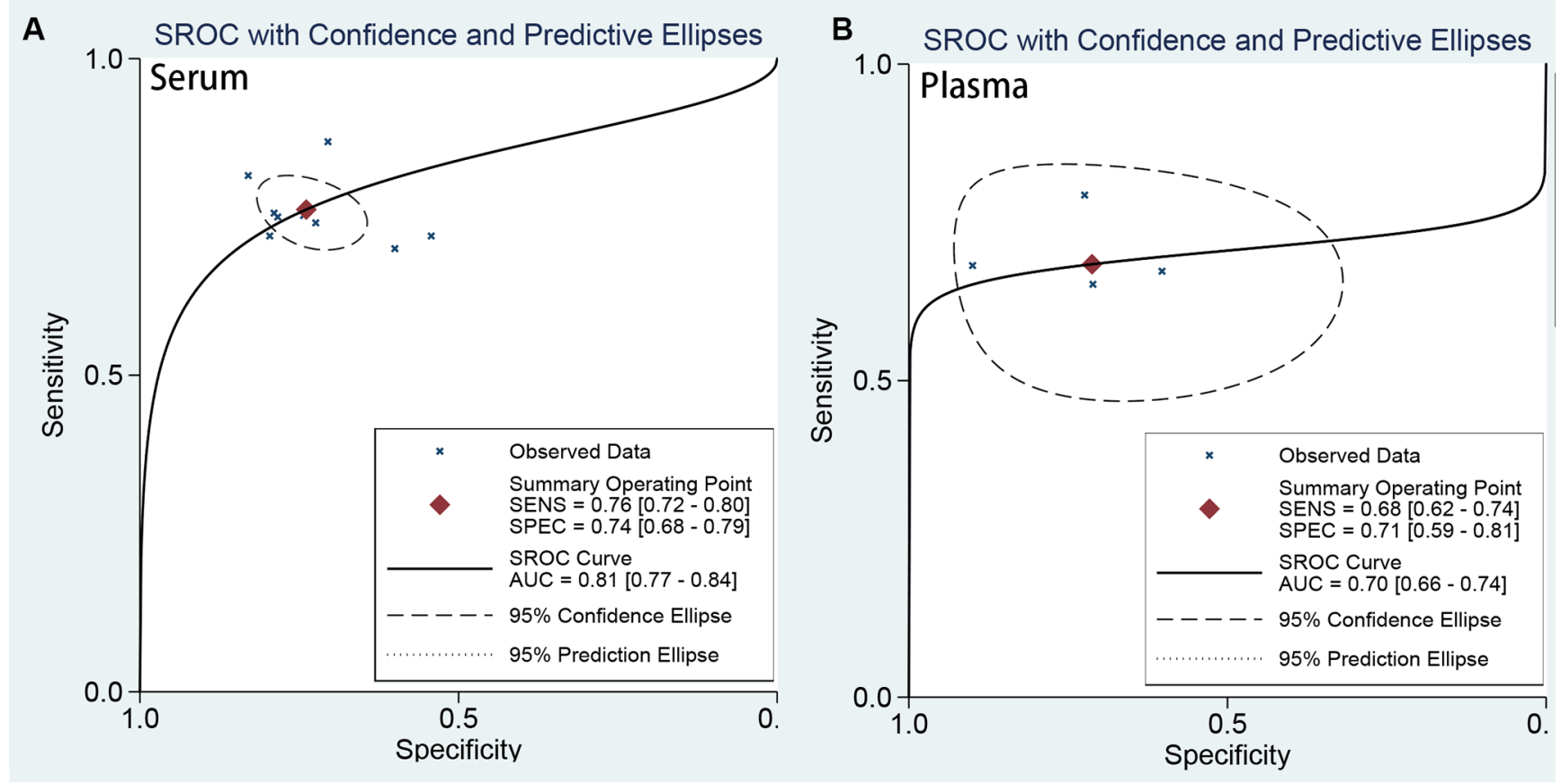

Figure 4: The SROC curve of miR-378 test for the diagnosis of various cancers (A) SROC curve of serum-based; (B) SROC curve of plasma-based). 
was reported to be increased in $\mathrm{GC}$ and $\mathrm{CRC}$ patients $[17,20]$. Not in parallel with these above results, Hause did not report the difference between RCC patients and controls $[18,24]$. Another study by Wang even found that the miRNA-378 levels were significantly decreased in the serum of RCC patients [25], and decreased levels were also found in patients with NPC (nasopharyngeal carcinoma) [16]. Many factors could be attributed to the results differences from three studies such as population selection, sample size and disease types. Some authors speculated that cancer cells could intentionally release or capture miRNAs, and lead to elevated or decreased miRNAs levels in tumor tissues. The biological effects depended on cell-specific collection in target genes. It is reported that miRNA-378 could inhibit human GC MDC- 803 cells by target MAPK1 in vitro, and promote BMP2-inducec osteogenic differentiation of mesenchymal progenitor cells [33]. Therefore, the single miRNA may have some limitations in detecting cancers, and the combination may provide more accurate diagnostic values.

The major strength of our study was that we strictly followed the PRISMA guidelines to conducted the metaanalysis, and evaluate the quality of include studies using the scale recommended by Cochrane Collaboration. There are still several limitations. First, in spite of the fact the present study yielded a moderate diagnostic value, we still recommend the combined miRNAs biomarkers to detect cancers. According to the criteria of high accuracy (PLR $>10$, NLR $<0.1$ ), the results of miR-378 are not high enough as expected. For clinical purpose, it is really necessary to make decisions combined with miRNAs. because it is reported that the combination of miRNA-371 with other miRNAs generate a more accurate result [18]. Second, there are seven types of cancer in the metaanalysis, and some cancer types are few, which makes our results more appropriate for detecting RCC. Further validation in large cohorts will be necessary. Third, the study subject of all include studies are Asian and Caucasian populations, and no studies with African population are included. The gene and mRNA could adjust the expression of protein. The mRNA could be an information carrier. The transcriptional regulation is the main way of gene expression, and post-transcriptional regulation also play an important role in the progression of gene expression. It is possible that different gene types could increase or decrease the expression of certain protein, which will lead to the occurrence of disease. Finally, our results showed that the diagnostic accuracy of serum-based specimen will be better than plasma-based in the overall cancers. Considering the different function of miRNAs, the value could be different in a specific caner type.

In conclusions, the overall diagnostic values of miR378 in the present meta-analyses are moderate accurate for human cancers, especially for RCC; The source of specimen has an effect on the diagnostic accuracy. The diagnostic value of serum-based was higher than that of plasma-based. The future study should focus on the mechanism and combined effect of miRNA-378 and others miRNAs.

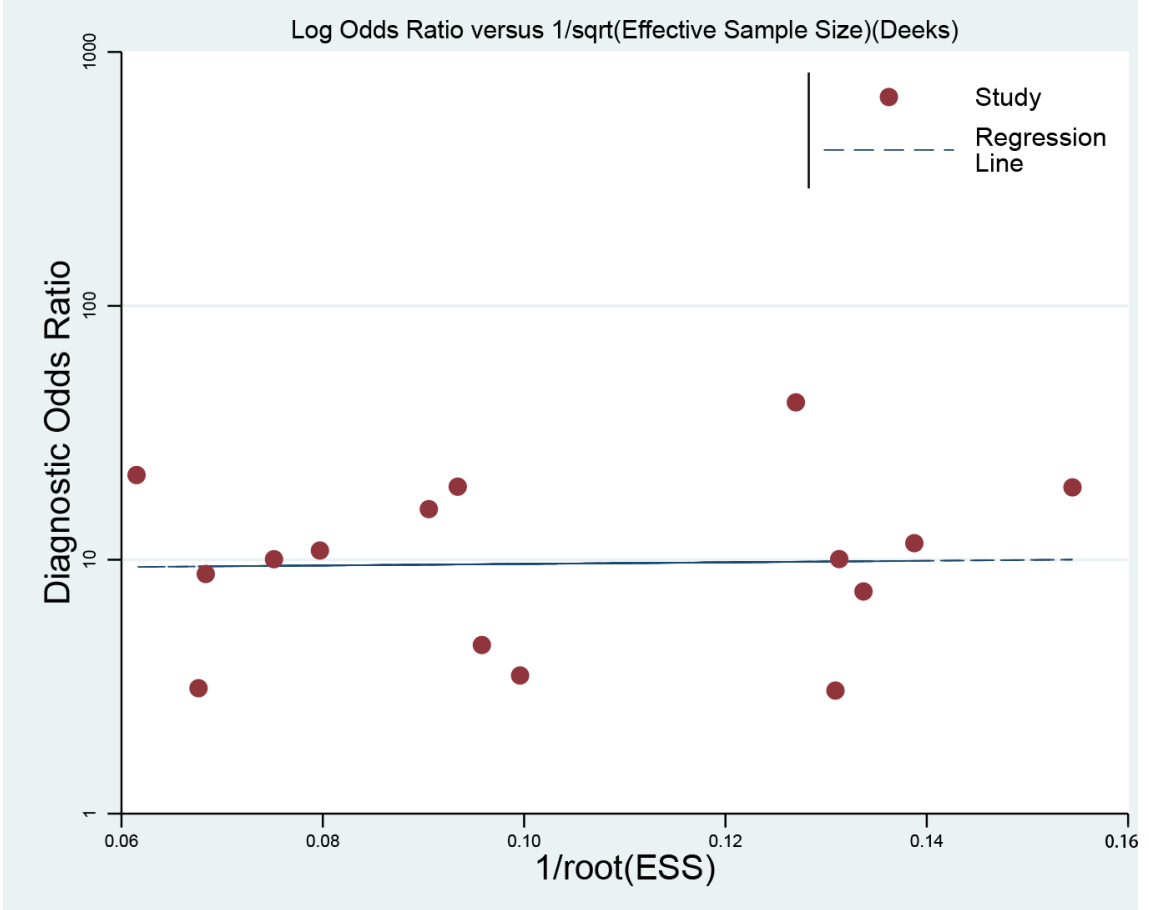

Figure 5: Deek's funnel plot to evaluate the publication bias. 


\section{MATERIALS AND METHODS}

The present meta-analysis followed the PRISMA Statement (Preferred Reporting Items for Systematic Reviews and Meta-Analyses Supplementary Table S1, Supplementary Table S3). The ethical approval is not necessary for the meta-analysis of the published studies [34].

\section{Literature search}

Systematic electronic searches were conducted in PubMed, Web of Science, Embase, CNKI (China National Knowledge Infrastructure), and Wanfang from the inception to January 15, 2016. We performed online searches using the possible Medical Subject Heading $(\mathrm{MeSH})$ terms and keywords. The following search terms were used: ('microRNA-378' OR 'miRNA-378' OR 'miR-378' OR 'has-mir-378') AND ('cancer' OR 'tumor' OR 'carcinoma' OR 'neoplasms') AND ('diagnostic' OR 'diagnoses' OR 'ROC curve' OR 'Diagnostic value' OR 'sensitivity' OR 'specificity' OR receiver operating characteristics). We also retrieved the reference lists of relevant articles and reviews to identify the potentially eligible studies. Our research was restricted to Chinese and English.

\section{Selection criteria}

Two researchers (ZZL and YYL) independently conducted the initial search, removed the duplicate records, screened the titles and abstracts forrecords, and identified records by scanning the full texts of publications. Any disagreements were resolved by fully discussion to consensus. Studies meeting the following criteria were included: (1) evaluated the diagnostic value of miR-378 for cancers; (2) Types of cancer in the studies were confirmed by gold standard. (3) Study could supply sufficient data for calculating four values (TP: true positives, FP: false positives, FN: false negatives, and TN: true negatives). Studies focused on other microRNAs, can't provide enough data were excluded. The latest studies were included for publications with duplicate data.

\section{Data extraction}

We used a standard sheet to collect relevant data. Data extraction was conducted by ZZL and checked independently by other two authors (LFS and PC). The following data were extracted: the first author, year of publication, country, ethnicity, sample size, type of cancer, source of sample, methods of detection, values of diagnostic 4-fold contingency table (TP, FP, FN, TN). We also tried to contact the authors of articles for the missing data, and resolved the discrepancies by discussing with other authors.

\section{Assessment of quality}

We used the Quality Assessment of Diagnostic Accuracy Studies 2(QUADAS-2) to assess the quality of included studies [35]. The QUADAS-2 tool consists of 4 key domains that discuss patient selection, index test, reference standard, and flow of patients through the study and timing of the index tests and reference standard (flow and timing). Each key domain includes two sections: risk of bias and applicability. If answers to all signaling questions for a domain are 'yes', then we could judge the risk of bias is low. If any question is answered 'no', potential bias exists. Concerns about applicability are judged as 'low', 'high', or 'unclear'.

\section{Statistical analysis}

First, we tested the threshold effect (Heterogeneity caused by adopting different diagnostic cutoff values when we conduct a meta-analysis of diagnostic test.) by calculating the Spearman correlation coefficient between sensitivity and specificity, If the threshold effect exists, we will combine the study results by fitting an ROC (Receiver Operating Characteristic) curve rather than pooling sensitivities and specificities or other index [36]. There is no threshold effect for the present study. We used the bivariate mixed effects models to estimate the combined sensitivity, specificity, PLRs (positive likelihood ratios), NLR (negative likelihood ratios), DORs (diagnostic odds ratios) and their 95\% CI (confidence intervals) [37]. We used Q test and $I^{2}$ to examine the heterogeneity qualitatively and quantitatively, respectively, and $I^{2}>50 \%$ presented the existence of heterogeneity [38]. We conducted subgroup analyses in the ethnicity (Asian vs Caucasian), cancer type (renal cell carcinoma vs other types), sample types (serum-based, plasma-based and) setting. We also calculated the area under the summary receiver operator characteristic (SROC) curve (AUC) with $95 \%$ CI. An AUC of 1.0 was judged as perfect diagnostic ability, and AUC $\leq 0.5$ presents a poor diagnosis [39]. Fagan plots shows the relationship between the prior probability, the likelihood ration, and posterior test probability, and Deek's funnel plot was used to evaluate the publication bias [40]. $P<0.05$ indicated statistically significant. All statistical analyses were performed on the Stata 12.0 station (Corp, College Station TX, USA) and Review Manager 5.3 (The Nordic Cochrane Centre, The Cochrane Collaboration, 2014.)

\section{Abbreviations}

AFP, $\alpha$-fetoprotein; CEA, carcinoembryonic antigen; CA, carbohydrate antigen; PRISM, Preferred Reporting Items for Systematic Reviews and Meta-Analyses; CNKI, China National Knowledge Infrastructure; TP, true 
positives; FP, false positives; FN, false negatives; TN, true negatives; ROC, Receiver Operating Characteristic; SROC, summary receiver operator characteristic PLRs, positive likelihood ratios; NLRs, negative likelihood ratios; DORs, diagnostic odds ratios; $\mathrm{CI}$, confidence intervals; RCC, renal cell cancer; $\mathrm{GC}$, gastric cancer; $\mathrm{NC}$, nasopharyngeal carcinoma; $\mathrm{CC}$, colorectal carcinoma; $\mathrm{BC}$, breast cancer; PC, pancreatic cancer. qRT-PCR, quantificational real-time polymerase chain reaction.

\section{ACKNOWLEDGMENTS AND FUNDING}

LZZ, SLF, and CLZ designed this study and contributed substantially to the design of the search strategy. LZZ and LYY searched and selected the trials and extracted data. PC performed the analysis and interpreted the data. LZZ and CLZ wrote the manuscript. LZZ and LYY critically reviewed the manuscript. LZZ, LYY and PC participated in the data extraction and critically revised it. LZZ and CLZ proofread the final version. All authors read and approved the final manuscript.

\section{CONFLICTS OF INTEREST}

The authors declare that they have no conflicts of interest.

\section{REFERENCES}

1. Ferlay J, Soerjomataram I, Dikshit R, Eser S, Mathers C, Rebelo M, Parkin DM, Forman D, Bray F. Cancer incidence and mortality worldwide: sources, methods and major patterns in GLOBOCAN 2012. Int J Cancer. 2015; 136:E359-386.

2. Gunes-Bayir A, Kiziltan HS, Senturk N, Mayadagli A, Gumus M. A Pilot Study of Self-Reported Physical Activity and Eating Habits in Turkish Cancer Patients Under Chemotherapy. Nutr Cancer. 2015; 67:906-911.

3. Rudolph A, Chang-Claude J, Schmidt MK. Geneenvironment interaction and risk of breast cancer. $\mathrm{Br} \mathrm{J}$ Cancer. 2016; 114:125-133.

4. Hang J, Cai B, Xue P, Wang L, Hu H, Zhou Y, Ren S, Wu J, Zhu M, Chen D, Yang H, Wang L. The Joint Effects of Lifestyle Factors and Comorbidities on the Risk of Colorectal Cancer: A Large Chinese Retrospective CaseControl Study. PLoS One. 2015; 10:e143696.

5. Breitkreutz D, Hlatky L, Rietman E, Tuszynski JA. Molecular signaling network complexity is correlated with cancer patient survivability. Proc Natl Acad Sci U S A. 2012; 109:9209-9212.

6. Fossa SD, Nilssen Y, Kvale R, Hernes E, Axcrona K, Moller B. Treatment and 5-year survival in patients with nonmetastatic prostate cancer: the Norwegian experience. Urology. 2014; 83:146-152.
7. Wang $\mathrm{P}$, Meng ZQ, Chen Z, Lin JH, Zhou ZH, Chen H, Wang K, Shen YH, Zhu ZF, Zhao GF, Fu H, Liu LM. Survival rate of pancreatic cancer in elderly patients. Hepatogastroenterology. 2008; 55:681-686.

8. El-Serag HB, Rudolph KL. Hepatocellular carcinoma: epidemiology and molecular carcinogenesis. Gastroenterology. 2007; 132:2557-2576.

9. Wang Q, Wu X, Wu T, Li GM, Shi Y. Clinical significance of RKIP mRNA expression in non-small cell lung cancer. Tumour Biol. 2014; 35:4377-4380.

10. Tran B, Rosenthal MA. Survival comparison between glioblastoma multiforme and other incurable cancers. J Clin Neurosci. 2010; 17:417-421.

11. Xiong K, Xue LX. The Application of Genomics and Epigenetics in Tumor Marker Research. Chinese Journal of Biochemistry \& Molecular Biology. 2014; 30:30-37.

12. Friedrich RE, Klapdor R, Bartel-Friedrich S. Rapidly progressive and metastatic mucoepidermoid carcinoma: application of serological tumor markers. Anticancer Res. 2007; 27:2099-2100

13. Garzon R, Marcucci G. Potential of microRNAs for cancer diagnostics, prognostication and therapy. Curr Opin Oncol. 2012; 24:655-659.

14. Berindan-Neagoe I, Monroig Pdel C, Pasculli B, Calin GA. MicroRNAome genome: a treasure for cancer diagnosis and therapy. CA Cancer J Clin. 2014; 64:311-336.

15. Ng EK, Chong WW, Jin H, Lam EK, Shin VY, Yu J, Poon TC, $\mathrm{Ng}$ SS, Sung JJ. Differential expression of microRNAs in plasma of patients with colorectal cancer: a potential marker for colorectal cancer screening. Gut. 2009; 58:1375-1381.

16. Liu X, Luo HN, Tian WD, Lu J, Li G, Wang L, Zhang B, Liang BJ, Peng XH, Lin SX, Peng Y, Li XP. Diagnostic and prognostic value of plasma microRNA deregulation in nasopharyngeal carcinoma. Cancer Biol Ther. 2013; 14:1133-1142.

17. Zanutto S, Pizzamiglio S, Ghilotti M, Bertan C, Ravagnani F, Perrone F, Leo E, Pilotti S, Verderio P, Gariboldi M, Pierotti MA. Circulating miR-378 in plasma: a reliable, haemolysis-independent biomarker for colorectal cancer. Br J Cancer. 2014; 110:1001-1007.

18. Redova M, Poprach A, Nekvindova J, Iliev R, Radova L, Lakomy R, Svoboda M, Vyzula R, Slaby O. Circulating miR-378 and miR-451 in serum are potential biomarkers for renal cell carcinoma. J Transl Med. 2012; 10:55.

19. Peng J, Xie Z, Cheng L, Zhang Y, Chen J, Yu H, Li Z, Kang H. Paired design study by real-time PCR: miR-378* and miR-145 are potent early diagnostic biomarkers of human colorectal cancer. BMC Cancer. 2015; 15:158.

20. Liu H, Zhu L, Liu B, Yang L, Meng X, Zhang W, Ma Y, Xiao H. Genome-wide microRNA profiles identify miR-378 as a serum biomarker for early detection of gastric cancer. Cancer Lett. 2012; 316:196-203. 
21. Yin JY, Deng ZQ, Liu FQ, Qian J, Lin J, Tang Q, Wen XM, Zhou JD, Zhang YY, Zhu XW. Association between mir-24 and mir-378 in formalin-fixed paraffin-embedded tissues of breast cancer. Int J Clin Exp Pathol. 2014; 7:4261-4267.

22. Fedorko M, Stanik M, Iliev R, Redova-Lojova M, Machackova T, Svoboda M, Pacik D, Dolezel J, Slaby O. Combination of MiR-378 and MiR-210 Serum Levels Enables Sensitive Detection of Renal Cell Carcinoma. Int J Mol Sci. 2015; 16:23382-23389.

23. Li A, Yu J, Kim H, Wolfgang CL, Canto MI, Hruban RH, Goggins M. MicroRNA array analysis finds elevated serum miR-1290 accurately distinguishes patients with low-stage pancreatic cancer from healthy and disease controls. Clin Cancer Res. 2013; 19:3600-3610.

24. Hauser S, Wulfken LM, Holdenrieder S, Moritz R, Ohlmann CH, Jung V, Becker F, Herrmann E, WalgenbachBrunagel G, von Ruecker A, Muller SC, Ellinger J. Analysis of serum microRNAs (miR-26a-2*, miR-191, miR-337-3p and miR-378) as potential biomarkers in renal cell carcinoma. Cancer Epidemiol. 2012; 36:391-394.

25. Wang C, Hu J, Lu M, Gu H, Zhou X, Chen X, Zen K, Zhang CY, Zhang T, Ge J, Wang J, Zhang C. A panel of five serum miRNAs as a potential diagnostic tool for early-stage renal cell carcinoma. Sci Rep. 2015; 5:7610.

26. Li D, Bo S, Shuangyang T, Peng C. Expression of plasma miR-210, miR-378 and theirs clinical significance in renal clear cell carcinoma. J Clin Urology. 2015; 30:897-901.

27. Shanshan L. (2013). Plasma miRNAs as Potential Biomarkers for Detecting Gastric Cancer. Anhui Medical University.

28. Stewart FA, Hoving S, Russell NS. Vascular damage as an underlying mechanism of cardiac and cerebral toxicity in irradiated cancer patients. Radiat Res. 2010; 174:865-869.

29. Shen L, Wan Z, Ma Y, Wu L, Liu F, Zang H, Xin S. The clinical utility of microRNA-21 as novel biomarker for diagnosing human cancers. Tumour Biol. 2015; 36:1993-2005.

30. Tang $\mathrm{K}, \mathrm{Xu} \mathrm{H}$. Prognostic value of meta-signature miRNAs in renal cell carcinoma: an integrated miRNA expression profiling analysis. Sci Rep. 2015; 5:10272.
31. Tian X, Chen Z, Shi S, Wang X, Wang W, Li N, Wang J. Clinical Diagnostic Implications of Body Fluid MiRNA in Oral Squamous Cell Carcinoma: A Meta-Analysis. Medicine (Baltimore). 2015; 94:e1324.

32. Dy LC, Buckel LJ, Hurwitz RM. Melanoma in situ with epidermal effacement: a compelling adjunctive finding. J Drugs Dermatol. 2007; 6:708-711.

33. Fei B, Wu H. MiR-378 inhibits progression of human gastric cancer MGC-803 cells by targeting MAPK1 in vitro. Oncol Res. 2012; 20:557-564.

34. Moher D, Liberati A, Tetzlaff J, Altman DG, Group P. Preferred reporting items for systematic reviews and meta-analyses: the PRISMA statement. PLoS Med. 2009; 6:e1000097.

35. Whiting PF, Rutjes AW, Westwood ME, Mallett S, Deeks JJ, Reitsma JB, Leeflang MM, Sterne JA, Bossuyt PM, Group Q-. QUADAS-2: a revised tool for the quality assessment of diagnostic accuracy studies. Ann Intern Med. 2011; 155:529-536.

36. Deville WL, Buntinx F, Bouter LM, Montori VM, de Vet HC, van der Windt DA, Bezemer PD. Conducting systematic reviews of diagnostic studies: didactic guidelines. BMC Med Res Methodol. 2002; 2:9.

37. Reitsma JB, Glas AS, Rutjes AW, Scholten RJ, Bossuyt PM, Zwinderman AH. Bivariate analysis of sensitivity and specificity produces informative summary measures in diagnostic reviews. J Clin Epidemiol. 2005; 58:982-990.

38. Higgins JP, Thompson SG, Deeks JJ, Altman DG. Measuring inconsistency in meta-analyses. BMJ. 2003; 327:557-560.

39. Hamza TH, Arends LR, van Houwelingen HC, Stijnen T. Multivariate random effects meta-analysis of diagnostic tests with multiple thresholds. BMC Med Res Methodol. 2009; 9:73.

40. Song F, Khan KS, Dinnes J, Sutton AJ. Asymmetric funnel plots and publication bias in meta-analyses of diagnostic accuracy. Int J Epidemiol. 2002; 31:88-95. 